Article

\title{
Dual-Functional Nanoscale Devices Using Phase-Change Materials: A Reconfigurable Perfect Absorber with Nonvolatile Resistance-Change Memory Characteristics
}

\author{
Niloufar Raeis-Hosseini ${ }^{1,+}(\mathbb{D})$ and Junsuk Rho ${ }^{1,2,3, *(\mathbb{D})}$ \\ 1 Department of Chemical Engineering, Pohang University of Science and Technology (POSTECH), \\ Pohang 37673, Korea; niloufar@postech.ac.kr \\ 2 Department of Mechanical Engineering, Pohang University of Science and Technology (POSTECH), \\ Pohang 37673, Korea \\ 3 National Institute of Nanomaterials Technology (NINT), Pohang 37673, Korea \\ * Correspondence: jsrho@postech.ac.kr \\ † Current address: Department of Electrical and Electronic Engineering, Imperial College London, \\ London SW7 2AZ, UK.
}

Received: 21 January 2019; Accepted: 6 February 2019; Published: 8 February 2019

\begin{abstract}
Integration of metamaterial and nonvolatile memory devices with tunable characteristics is an enthusing area of research. Designing a unique nanoscale prototype to achieve a metasurface with reliable resistive switching properties is an elusive goal. We demonstrate a method to exploit the advantages of a phase-change material (PCM) as a metamaterial light absorber and a nanoscale data storage device. We designed and simulated a metamaterial perfect absorber (MPA) that can be reconfigured by adjusting the visible light properties of a chalcogenide-based PCM. The suggested perfect absorber is based on a $\mathrm{Ge}_{2} \mathrm{Sb}_{2} \mathrm{Te}_{5}$ (GST) film, and is tuned between two distinct states by heat treatment. Furthermore, we fabricated and characterized a resistive switching memory (ReRAM) device with the same features. The MPA/ReRAM device with a conventional metal/dielectric/metal structure $\left(\mathrm{Ag} / \mathrm{GST} / \mathrm{Al}_{2} \mathrm{O}_{3} / \mathrm{Pt}\right.$ ) consisted of arrays of $\mathrm{Ag}$ squares patterned on a GST thin film and an alumina-coated Pt mirror on a glass substrate. Based on the numerical data, amorphous GST showed perfect absorbance in the visible spectrum, whereas, crystalline GST showed broadband perfect absorbance. The fabricated ReRAM device exhibited uniform, bidirectional, and programmable memory characteristics with a high ON/OFF ratio for nonvolatile memory applications. The elucidated origin of the bipolar resistive switching behavior is assigned to the formation and rupture of conductive filaments.
\end{abstract}

Keywords: metamaterial; phase-change material; memory; tunable; perfect absorber; $\mathrm{Ge}_{2} \mathrm{Sb}_{2} \mathrm{Te}_{5}$

\section{Introduction}

Use of phase-change materials (PCMs) in electrical and optical systems has introduced widespread applications, including uses in optical data storage [1-3] and photonic nanodevices [4,5]. Attributes of PCMs include nonvolatile phase changes, rapid switching, and quick responses to stimuli, reasonable data retention, and long-term stability [6-8]. The exceptional properties of PCMs, including phase-state stability, small crystallization times $(\sim \mathrm{ns})$, low switching energies $(\sim p J)$, and high endurance potentials $\left(\sim 10^{15}\right.$ cycles), make them perfect materials for ultrafast and low-energy photonic processing [9]. Due to various physical properties of their atomic structures [10], PCMs can work as data storage systems in optical storage discs [11], emerging nonvolatile electronic memories (eNVMs) [6], active optoelectronic nanodisplays [12], and electro-optomechanics [13]. 
$\mathrm{Ge}_{2} \mathrm{Sb}_{2} \mathrm{Te}_{5}$ (GST) is a chalcogenide PCM that has been used for both nanoelectronic and nanophotonic systems. GST has unique characteristics in terms of both electrical (resistivity) and optical properties, like the refractive index (RI) between its crystalline and amorphous states [14-16]. The transitions between the two states are attained by localized heating, using optical or electrical energy [14,17]. Crystalline GST shows higher reflectivity and electrical conductivity than amorphous GST [17]. Recently, a photonic abacus [18] and brain-like computing [19] were realized by exploiting this difference in the properties of crystalline and amorphous GST [16]. The photonic memories using other materials show a volatile behavior [20,21]; however, a durable and nonvolatile GST-based photonic memory can realize eight-level bit storage in a single device by switching among intermediate states [22].

An external stimulus, such as an electrical pulse or thermal excitation from an elevated temperature above the transition temperature of GST, causes crystallization of as-deposited amorphous PCM. In contrast, another thermal stimulus with a higher magnitude during a shorter time (in the nanosecond range) will return the PCM to its initial state [23].

Absorbers based on metamaterials (MM), which can be used to manipulate light [24], have good flexibility [25] and are easily fabricated on a chip [26]. MM absorbers consist of an artificial arrangement of subwavelength metallic constructions on a dielectric-coated metal film; they also have unconventional properties [27]. Wide-band MM perfect absorbers [28], multiband absorbers [29,30], and polarization-dependent absorbers have been studied using various materials [31]. MMs with diverse resonances have been used to fabricate broadband absorbers [32,33].

To realize a reconfigurable MM, the geometry must be controlled and proper materials for dielectric layers and metallic mirrors are required [34]. Metamaterial perfect absorbers (MPAs) have a near perfect absorption $A$ and an absorption frequency that can be controlled by adjusting the MPA's permittivity $\varepsilon$, permeability $\mu$, and dimensions of metallic patterns in artificially periodic structures [35].

The main factors to be considered during the design of MM absorbers are their polarization dependencies, incidence angles, and bandwidths [16]. Perfect absorbers are the most favorable category of the MM absorbers that have been extensively examined in infra-red (IR) regimes [36]. Achieving a broadband absorbance in the visible domain is a useful goal [37], so we investigate an MPA in visible frequencies. Furthermore, the perfect absorption of electromagnetic (EM) waves is the most desirable characteristic of the developed MM absorbers [16]. The imaginary part of dielectric permittivity in the visible range is high in GST, so we expect a high $A$ in this regime. The high RI leads to a rapid transition between the phases of GST, and an increase in its wideband absorbance [37]. Previous work has demonstrated switchable metasurfaces [38], tunable nanophotonics [39], all-optical memories [26], and photonic calculators [18]. We extend this progress to develop a GST metadevice for use as resistance-change memory.

Resistive switching memory (ReRAM) has emerged as a potential candidate for the next generation of nanoelectronic devices because it is nonvolatile, has a high density, and is scalable [40]. Various materials have been reported as active layers of the ReRAM, including chalcogenides [6], oxides, and organic materials [41]. In this study we investigated a metamaterial perfect absorber with a hybrid oxide PCM layer to get an understanding of the assimilated materials used in attaining a reproducible and reliable ReRAM device.

We propose an MPA that has a metal-insulator-metal (MIM) structure with GST as a high-dielectric material on an $\mathrm{Al}_{2} \mathrm{O}_{3}$-coated $\mathrm{Pt}$ layer on a glass substrate and under an electrode that bears a square-patterned array of $\mathrm{Ag}$. The Pt bottom electrode works as a mirror to eliminate transmittance, and the Ag squares act as plasmon resonators. The resonance conditions of the MPA are manipulated by adjusting the thickness of a bilayer spacer (BS) made of GST and $\mathrm{Al}_{2} \mathrm{O}_{3}$. The BS generates magnetic resonance from the coupling between $\mathrm{Ag}$ and Pt electrodes [37]. To demonstrate the resistance change properties, we fabricate and electrically characterize a nanodevice with the same structure to realize a ReRAM with dual functionality. 


\section{Methods}

\subsection{Fabrication of the Memory Device}

ReRAM samples with MIM structures of $\mathrm{Ag} / \mathrm{GST} / \mathrm{Al}_{2} \mathrm{O}_{3} / \mathrm{Pt}$ were fabricated on a quartz glass substrate. The glass substrates were cleaned ultrasonically using acetone, 2-propanol, and distilled water for 15 min sequentially, then dried by blowing $\mathrm{N}_{2}$ gas. A $10 \mathrm{~nm}$ Ti adhesion layer was deposited on the substrate by E-beam evaporation, then a $100 \mathrm{~nm}$ Pt mirror was deposited on it by sputtering. A $7 \mathrm{~nm} \mathrm{Al}{ }_{2} \mathrm{O}_{3}$ thin film was deposited on the Pt electrode by using plasma-enhanced atomic layer deposition (PEADL) (Quros, Plus 200). A $25 \mathrm{~nm}$ GST layer was sputtered using RF magnetron sputtering (SnTek) with a background pressure of $5 \mathrm{mTorr}$ and a power of $50 \mathrm{~W}$ for $143 \mathrm{~s}$ under ambient gas.

Square nanopatterns of the MPA were defined using electron beam lithography (EBL) and wet chemical lift-off. To make the squares, PMMA 495 A4 was spin-coated at 4000 RPM, and then baked at $180^{\circ} \mathrm{C}$ for $2 \mathrm{~min}$. After EBL, an $80 \mathrm{~nm}$ Ag layer was deposited by E-beam evaporation on the lithographically patterned E-beam layer. A lift-off process was performed using acetone, followed by a 1 min ultrasonication process.

\subsection{Characterizations}

Conductive atomic force microscopy (Park systems, X100) using Pt-/Ir-coated tips was performed to measure the current-voltage $(I-V)$ characteristics of MPA. Scanning electron microscope (SEM) images were obtained with a Jeol 6500F SEM at $10 \mathrm{kV}$ for characterization of the fabricated device.

\subsection{Numerical Simulations}

The simulation with a full-field electromagnetic calculation was performed using Lumerical analysis based on the finite-difference time-domain method (FDTD), with normal incident light to the $x-y$ plane on the unit cell of MPA, and the E-field polarized in the $x$ direction.

A unit cell of MPA was simulated by periodic boundary conditions along the $x$ and $y$ axes. Perfectly matched layers were considered along with the propagation of the EM wave on the $z$ axis. The periodic property of the MPA was accounted for at $x= \pm \mathrm{L} / 2$ and $y= \pm \mathrm{L} / 2$ for the electric and magnetic conductor.

The optical constants and dielectric properties of $\mathrm{Ag}, \mathrm{Pt}$, and $\mathrm{Al}_{2} \mathrm{O}_{3}$ were selected from the available Lumerical library. The dielectric properties of GST in two different phases were calculated from the ellipsometry data.

\section{Results and Discussion}

\subsection{Structure and Design}

A nanoscale device with a sandwiched structure configuration of resonator/top spacer layer/bottom spacer layer/mirror (i.e., Ag nanopatterns/GST $/ \mathrm{Al}_{2} \mathrm{O}_{3} / \mathrm{Pt}$ ) (Figure 1a-c) was used to demonstrate the MPA and ReRAM. A $100 \mathrm{~nm}$ Pt electrode with a $5 \mathrm{~nm} \mathrm{Cr}$ adhesion layer was deposited as a bottom electrode (BE) on a glass substrate. A $7 \mathrm{~nm} \mathrm{Al} \mathrm{O}_{3}$ layer was ALD-deposited onto the Pt electrode, then covered with a $25 \mathrm{~nm}$ GST film by sputtering (Figure 1b). Ag squares with side lengths $(\mathrm{d} x, \mathrm{~d} y)$ of $110 \mathrm{~nm}$, a thickness of $80 \mathrm{~nm}$, and a lattice constant of $220 \mathrm{~nm}$ (Figure $1 \mathrm{~b}$ ) were patterned on a GST layer using e-beam lithography (EBL), then deposited using e-beam evaporation. SEM images of the fabricated MPA, with $5 \times 5$ arrays of the nanostructure with different magnifications (Figure 2a-d) and a cross-sectional SEM image (Figure S1a), confirmed successful fabrication of the device. Atomic force microscopy (AFM) confirmed that $110 \mathrm{~nm} \mathrm{Ag}$ patterns were well-formed on the GST thin film (Figure 2e,f). The cross-sectional SEM image of the fabricated device illustrated the well-fabricated ReRAM (Figure S1a in the Supplementary Materials). 


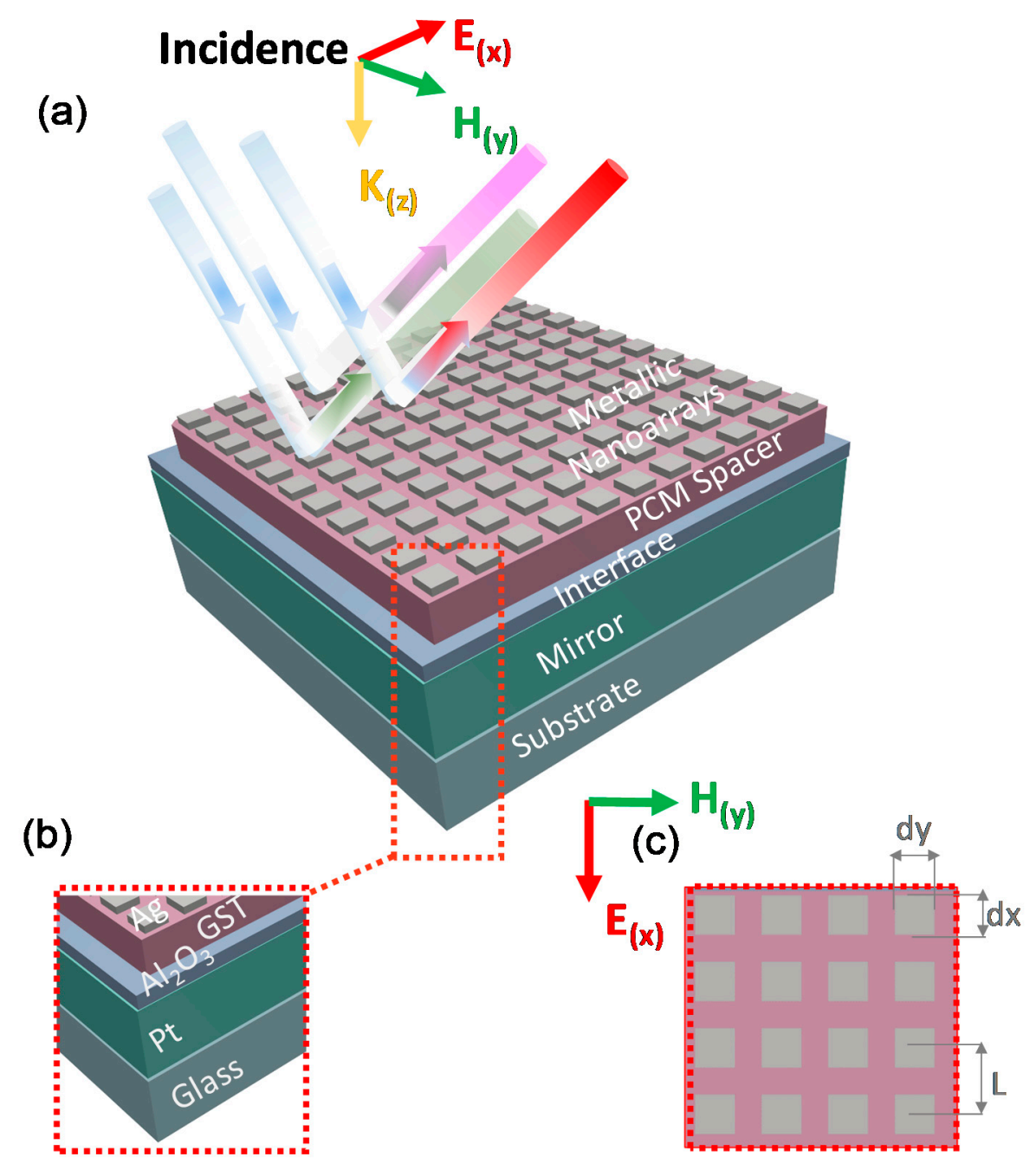

Figure 1. (a) Illustration of the reconfigurable and programmable metamaterial perfect absorber (RPMPA) and resistive switching memory (ReRAM) structures, including the incident light polarization conformation. Glass (silica) was selected as a substrate with a thickness of $250 \mu \mathrm{m}$. (b) Schematic of a unit cell of the RPMPA and ReRAM with an $\mathrm{Ag} / \mathrm{Ge}_{2} \mathrm{Sb}_{2} \mathrm{Te}_{5}$ (GST) $/ \mathrm{Al}_{2} \mathrm{O}_{3} / \mathrm{Pt} / \mathrm{SiO}_{2}$ structure. (c) Top view of the RPMPA and ReRAM, and the Ag nanosquares with $\mathrm{d} x$ and $\mathrm{d} y$ dimensions and chips of $L$ (the middle to middle distance between the patterned squares).

\section{2. $\mathrm{Ge}_{2} \mathrm{Sb}_{2} \mathrm{Te}_{5}$ (GST) Thin Film Characterization}

Samples were characterized following the sputtering of the GST layer and heating above the transition temperature. AFM analysis confirmed that amorphous GST formed a smooth, thin layer. The heated GST thin film changed to the crystalline phase, with a consequent increase in surface roughness compared to the amorphous state (Figure S2a,b). Interaction of the $\mathrm{Pt}$ mirror with the $\mathrm{Ag}$ squares localized the electromagnetic (EM) field inside the BS layer.

\subsection{Optical Properties of GST}

PCMs were characterized by their amorphous and crystalline states related to covalent and resonant bonding, respectfully. The phase transition between two metastable states was nonvolatile [34]. 
(a)

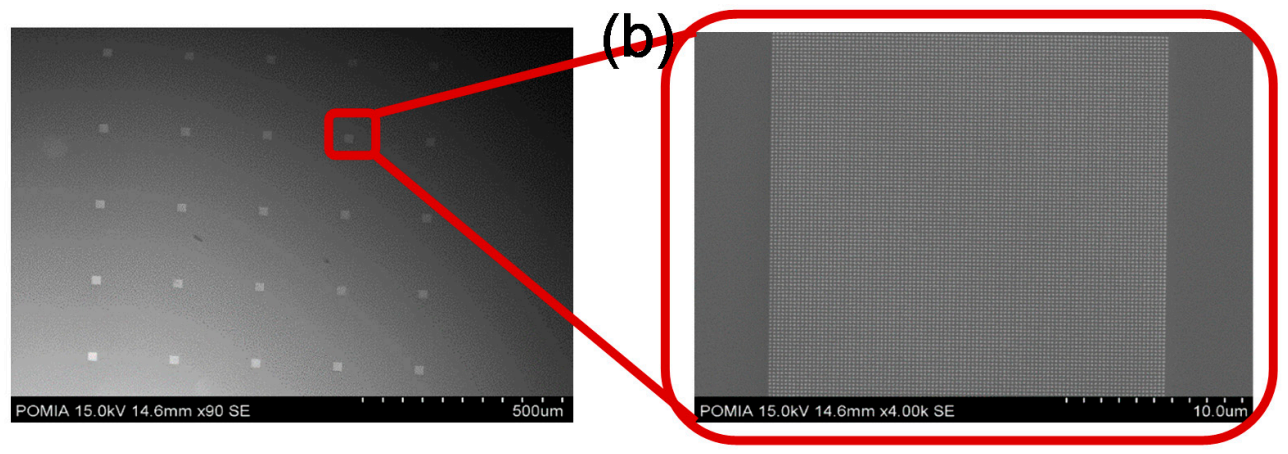

(c)

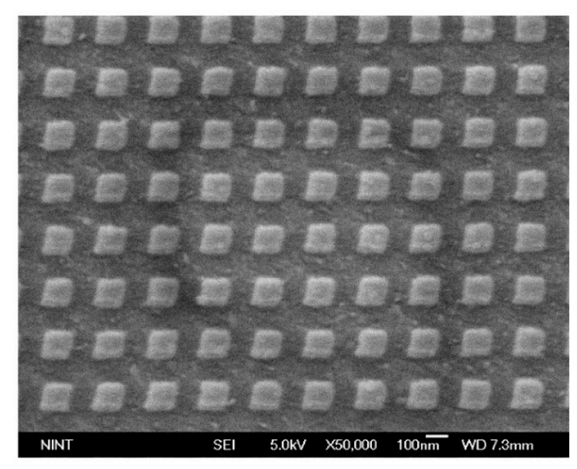

(d)

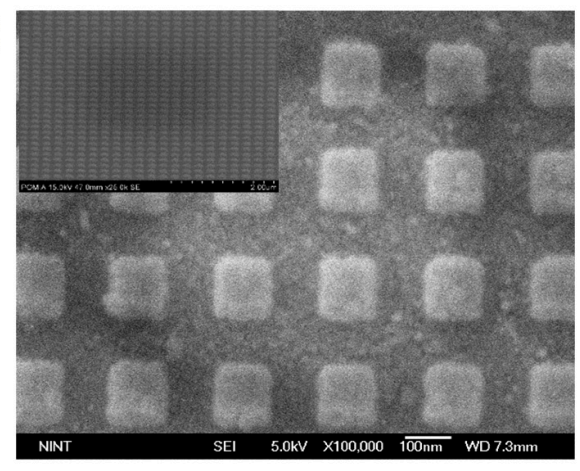

(e)

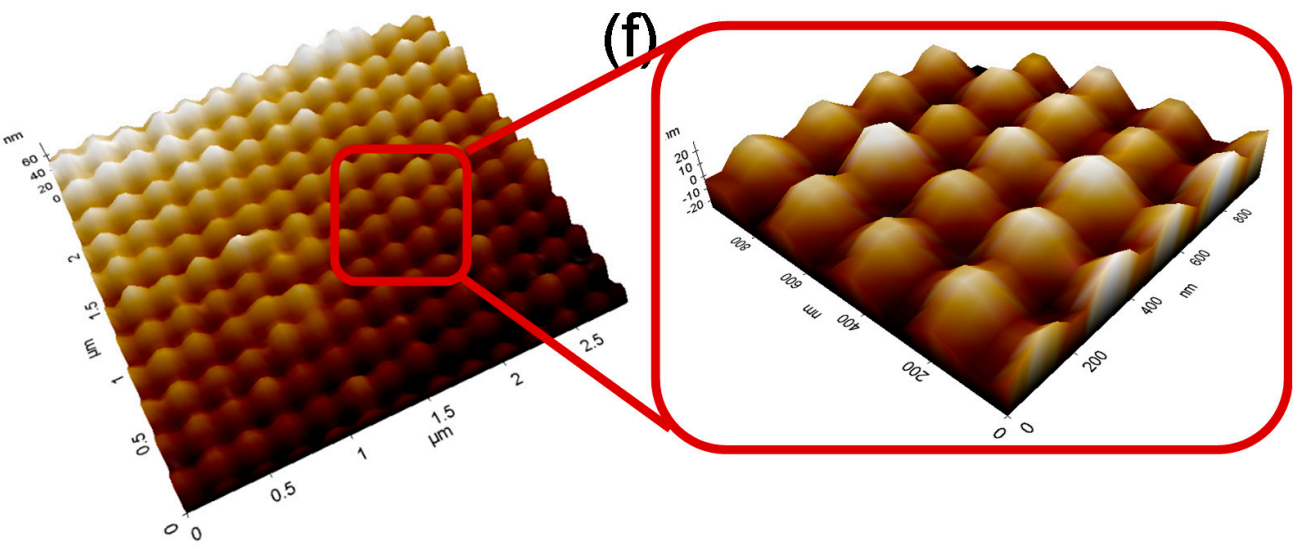

Figure 2. (a) Scanning electron microscope (SEM) images of the fabricated resistive switching memory (ReRAM) and metamaterial perfect absorber (MPA) devices with $5 \times 5$ arrays of nanostructures; (b) A magnified image of one square patch with dimensions of $100 \mu \mathrm{m}$; (c) $120 \times 120 \mathrm{~nm}$ patterned square-shaped nanostructures; and (d) $20^{\circ}$ tilted square patterns; inset: a perspective view of the ReRAM. (e) AFM image of the patterned Ag top electrode and (f) the magnified AFM image.

The refractive index $n$ and extinction coefficient $k$ of amorphous and crystalline GST were obtained from ellipsometry data in the ultraviolet (UV) spectral range up to a wavelength of $\lambda=1000 \mathrm{~nm}$ (Figure $3 \mathrm{a}, \mathrm{b}$ ). In the amorphous state, the predominant peaks of $n$ and $k$ were recorded in the visible range $(\sim 400 \mathrm{~nm})$; however, a smaller second peak of $k$ was observed at $300 \mathrm{~nm}$.

Although the maximum $n$ of crystalline state occurred at $400 \mathrm{~nm}$, different peaks of $k$ (at 0.6 and 0.55 ) happened in UV ranges (about 200, 300, and $380 \mathrm{~nm}$ ) following a peak of $0.45 \mathrm{in}$ NIR (>800 nm) regions. Upon crystallization, the temperature $n$ increased from 1.4 to 1.7 at approximately $400 \mathrm{~nm}$ (Figure 3a); however, $k$ was enhanced by about 45 times at a wavelength of $820 \mathrm{~nm}$ (Figure $3 b$ ).

A significant change in bonding was responsible for the pronounced increase in the optical properties associated with attenuation in a bandgap [42]. We concluded that the dielectric constant was dispersive [37] and that the large, imaginary part of the dielectric constant of GST indicated a high $A$ [43]. 
(a)

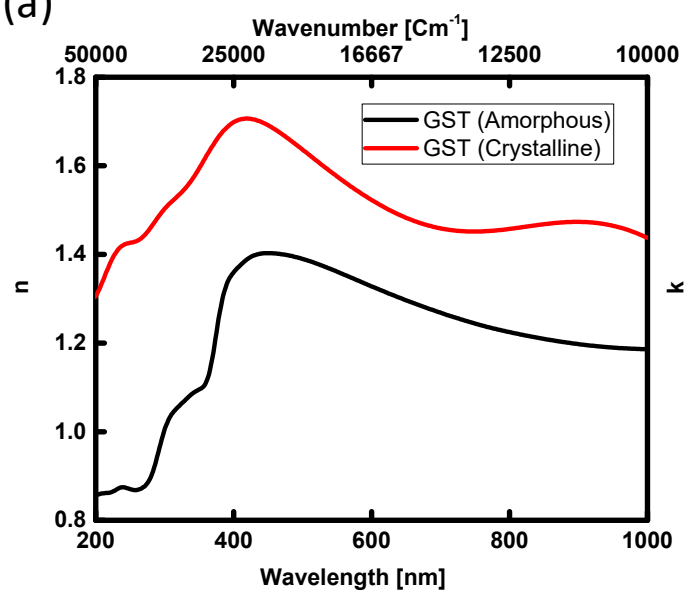

(b)

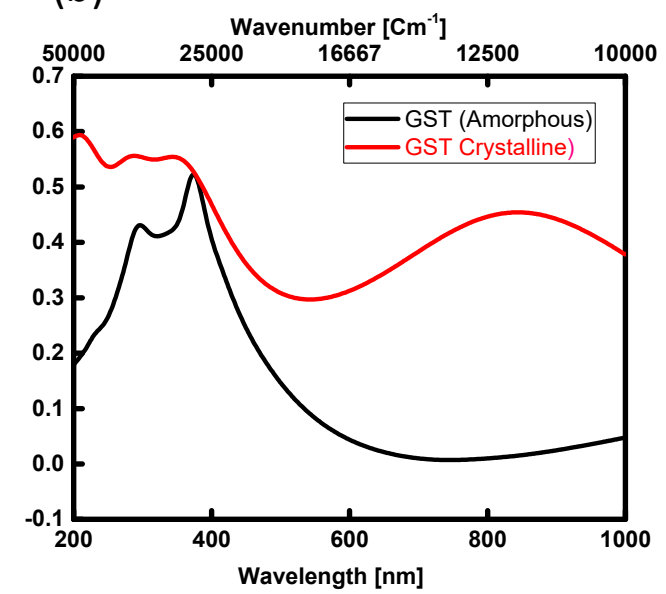

Figure 3. Dielectric constant (a) $n$ and (b) $k$ in the $200 \leq \lambda \leq 1000 \mathrm{~nm}$ wavelength range for the amorphous and crystalline phases of $\mathrm{Ge}_{2} \mathrm{Sb}_{2} \mathrm{Te}_{5}$, obtained from ellipsometry data.

\subsection{Simulation Data of the Metamaterial Perfect Absorber (MPA)}

The MPA was simulated using the finite-difference time-domain method (FDTD) with a transverse electric (TE) plane wave. To design a device with $A=1$, the dimensions and structural parameters were optimized by simulation. In the FDTD simulation, a source was selected within the visible range with a downward propagation along the $z$ axis, whereas the electric field $E$ was polarized in the $x$ direction. Preliminary simulations were performed to optimize the thickness of the $\mathrm{Al}_{2} \mathrm{O}_{3}$ interface layer and the GST thin film, and to optimize the dimensions of Ag nano-squares. Based on the achieved results, we fixed the thicknesses of GST and $\mathrm{Al}_{2} \mathrm{O}_{3}$ at 25 and $7 \mathrm{~nm}$, respectively. The absorbance over the visible range of the spectrum was simulated for the amorphous GST-based metadevice with different thicknesses $\left(t_{\mathrm{Ag}}\right)$ of the top Ag electrode $(60,80,100,140,180$, and $220 \mathrm{~nm}$ ) (Figure 4a). A thickness of $t_{\mathrm{Ag}}=60 \mathrm{~nm}$ yielded an $A$ of about 0.98 , and a thickness of $80 \mathrm{~nm} \leq t_{\mathrm{Ag}} \leq 220 \mathrm{~nm}$ yielded an $A=$ 1.0 at $560 \leq \lambda \leq 690$ (Figure 4a). Therefore, we selected the minimum $t_{\mathrm{Ag}}$ that achieved an $A=1.0$ (i.e., $t_{\mathrm{Ag}}=80 \mathrm{~nm}$ for $\lambda=560 \mathrm{~nm}$, and $t_{\mathrm{Ag}}=100 \mathrm{~nm}$ for $\lambda \sim 600 \mathrm{~nm}$ ). By using GST film in a crystalline state, a broadband perfect absorber was achieved for $600 \leq \lambda \leq 900 \mathrm{~nm}$ (Figure $4 \mathrm{~b}$ ). The Ag squares governed the electric dipole resonances, and the Pt layer controlled the magnetic dipolar resonances. The simulation results confirmed that the dimensions and thickness of the nanopatterns, and thickness of the BS, are important factors that affect absorbance [37].

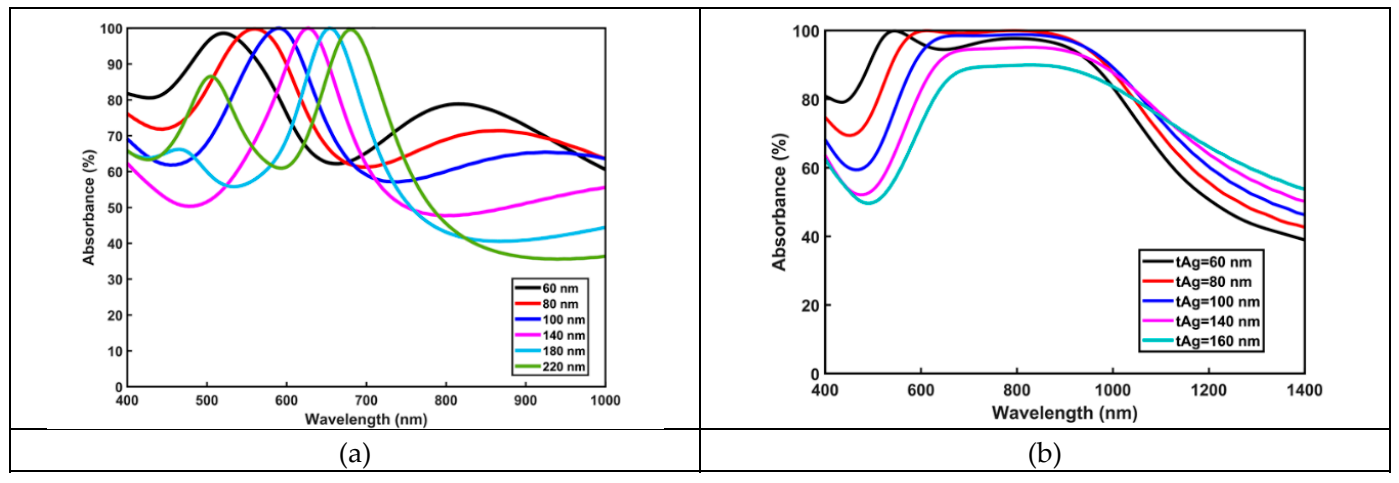

Figure 4. Metamaterial perfect absorbance (MPA) effect by finite-difference time-domain (FDTD) simulation. Reconfigurable perfect absorbance for (a) amorphous GST by changing the thickness of Ag nanoarrays from 60 to $220 \mathrm{~nm}$. (b) Broadband MPA effect using crystalline GST at Ag thicknesses from 60 to $160 \mathrm{~nm}$. 
The narrow absorption peaks of the MPA with amorphous GST were changed to broadband absorbance when the GST was crystalline. Perfect absorbance was likely to be caused by plasmonic resonance. Due to the matching impedance of MPA with the vacuum, low reflectance and high absorbance over the entire visible domain were achieved by using a crystalline GST layer. Adjustments of resonances in $\varepsilon$ and $\mu$ matched the impedance of the MPA to that of free space, producing an effective absorber [44].

To investigate the effect of BS on the ability to achieve perfect absorbance, and to gain insight into the importance of the localized surface plasmon resonance (LSPR), the electromagnetic distribution was calculated at a normal incidence (i.e., in the $x-z$ plane) (Figure $5 \mathrm{a}-\mathrm{d}$ ). The simulation considered Ag metallic squares with sides of $110 \mathrm{~nm}$ and a pitch of $220 \mathrm{~nm}$ under periodic conditions. Absorbance was calculated from the electric field as

$$
A=\frac{1}{2} \operatorname{Re}\left(-i \omega \cdot \varepsilon \cdot \varepsilon_{0} \cdot E^{*} \cdot E\right),
$$

where $\varepsilon$ and $\varepsilon_{0}$ are the permittivity of the material and free space, respectively. The electric field, magnetic field, and absorption were calculated for $\lambda \approx 610 \mathrm{~nm}$ (Figure $5 \mathrm{~b}-\mathrm{d}$ ).
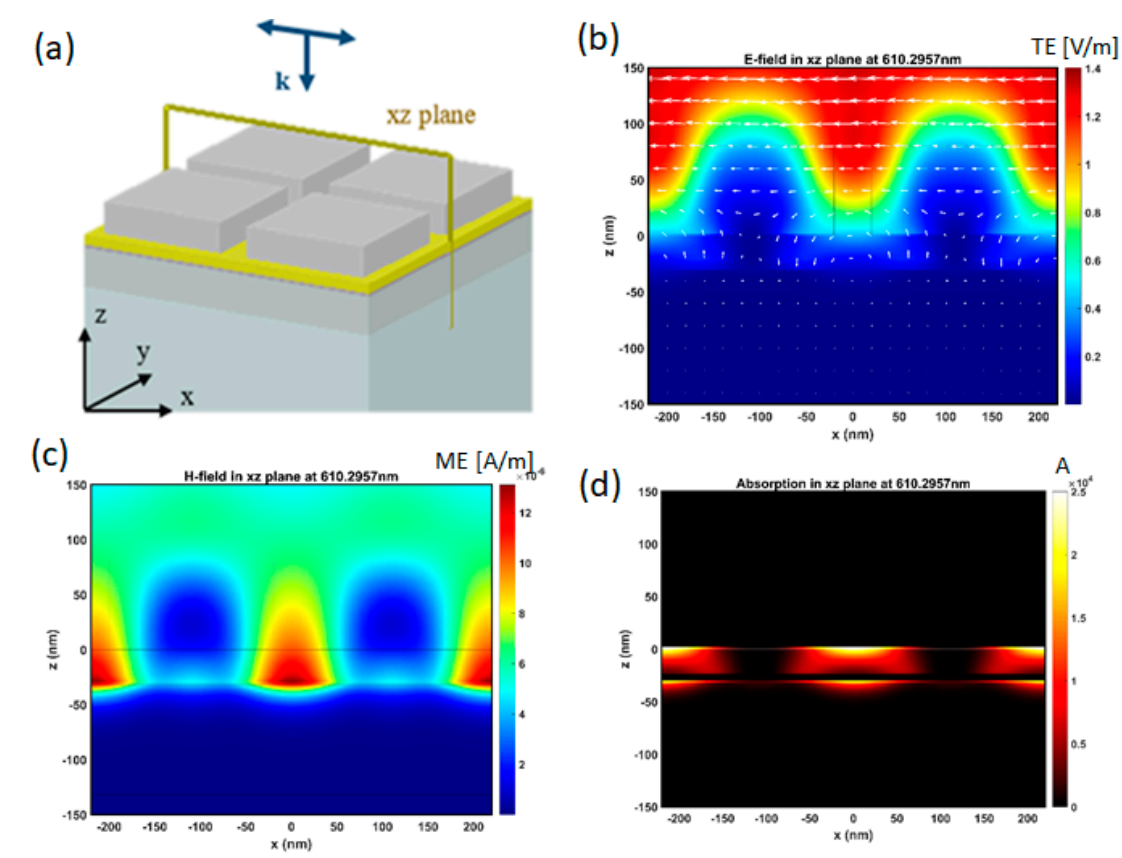

Figure 5. (a) Three-dimensional schematic view of the MPA along the $x-z$ plane. Simulated (b) electric field, (c) magnetic field, and (d) absorption in the $x-z$ plane for $\lambda \approx 610 \mathrm{~nm}$.

Two resonances were represented: one was an electric dipole between upper parts of Ag squares, the other was a magnetic dipole between their lower parts. The simulations suggest that electric fields responsible for the energy absorption through the BS layer propagated towards the base of the square patterns in a forward direction (Figure $5 b$, white arrows). Similar radiation of an individual electric dipole in two opposite directions (perpendicular to the dipole's orientation) caused an equal energy distribution. The electric and magnetic dipoles interfered destructively in the backward direction and constructively in the forward direction so that the backward scattering was suppressed while the forward scattering was strengthened $[45,46]$. This phenomenon led to a unidirectional super-scattering, or the so-called Kerker scattering [47]. The strengthening of the forward scattering and the entire absorption by the PCM layer combined to yield $A=1.0$.

The strong intensity of the magnetic field in the central part between the two unit cells represented a powerful magnetic dipolar moment between them [48]. 


\subsection{Fast Phase Transition of GST in the MPA}

Absorption happens because of losses within the BS layer. GST has a large imaginary part of the refractive index (Figure $3 \mathrm{~b}$ ), so a strong absorption peak in the visible range can be predicted. The high $A$ leads to a rapid increase in the GST temperature; therefore, the GST phase changes rapidly (nanosecond timescale) from amorphous to crystalline. ${ }^{37}$ The thermal energy $(\mathrm{J})$ that a unit cell can absorb is

$$
A=L^{2} \times F_{1} \times R_{a}
$$

where $E_{t h}, L$, and $F_{1}$ represent the thermal energy, mathematical lattice constant, and the light fluence shining on the sample, respectively. $R_{a}$ can be calculated from the overlap of light-power density and the absorbance spectrum within the visible range [37].

\subsection{Electrical Properties of Hybrid GST-Based Resistive Switching Memory (ReRAM)}

ReRAM is a possible novel memory device for future data storage systems; therefore, we characterized the electrical properties of the fabricated device to make it suitable for storing data. The GST-based ReRAM showed resistive switching behavior (Figure 6a). The current-voltage relation of the $\mathrm{Ag} / \mathrm{GST} / \mathrm{Al}_{2} \mathrm{O}_{3} / \mathrm{Pt}$ device was obtained with direct current voltage applied to the Ag electrode while the Pt electrode was grounded. Triggering the device by applying forming voltage caused the formation of two conductive filaments (CF), one composed of Ag within the GST layer and one composed of oxygen vacancies within the $\mathrm{Al}_{2} \mathrm{O}_{3}$ layer. After the $\mathrm{CFs}$ had formed, the insulating layers changed to their low-resistance state (LRS). We applied a compliance current of $10^{-4} \mathrm{~A}$ to prevent permanent breakdown of the device. In contrast, a change to the polarity of the applied voltage caused the $\mathrm{CF}$ to rupture, so insulating layers changed to their high-resistance state (HRS) (Figure 6a).

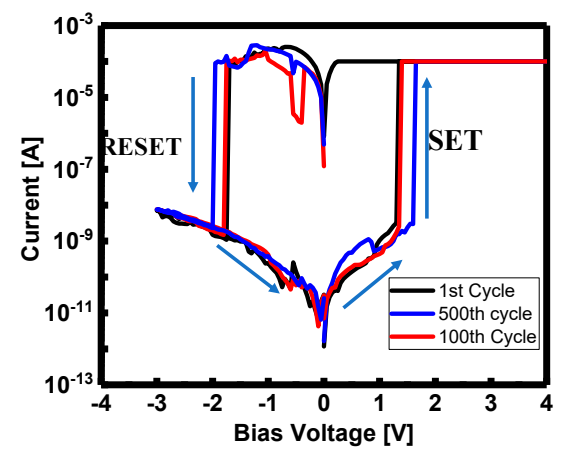

(a)

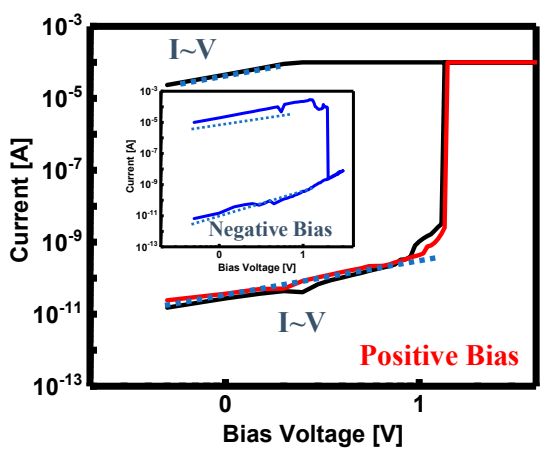

(b)

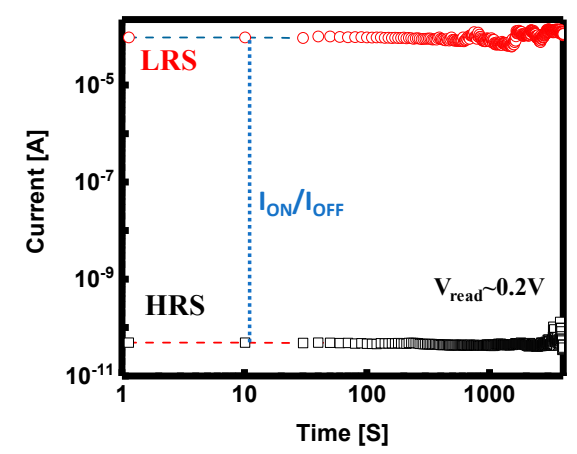

(c)

Figure 6. (a) Semi-logarithmic $I-V$ characteristics of the resistive switching memory device (ReRAM) with bilayer insulator film for 100 cycles. (b) Double-logarithmic plot for Ag/GST $/ \mathrm{Al}_{2} \mathrm{O}_{3} / \mathrm{Pt} / \mathrm{SiO}_{2}$ in the positive and negative sweep with Ohmic model. (c) Data retention characteristics of the low-resistance state (LRS) and high-resistance state (HRS) under continuous readout voltages for the as-fabricated ReRAM device. 
A repeated cyclic test was performed for more than 100 cycles; results confirmed that switching in the device was reproducible. Measurements of 100 cycles demonstrated that bipolar switching did not affect the electrical properties of the device (Figure 6a). A semilogarithmic $I-V$ curve (Figure 6a) suggested that the changes in conductivity were consequences of formation and rupture of CFs.

Double-logarithmic plots of the $I-V$ curves during positive and negative voltage sweeps confirmed the construction of CFs inside the insulator layer. Furthermore, the equations of the fitted curves were Ohmic for both LRS and HRS ( $\alpha$ $\alpha$ ) (Figure 6b). Fitting the curves to the results of positive and negative biases indicated that Ohmic conduction was the dominant phenomenon at both low and high voltages [6].

To determine the reliability of the fabricated device, the data retention test was performed. We examined the ReRAM device with a reading voltage of $0.2 \mathrm{~V}$. For both LRS and HRS states, the stored data were retained without any noticeable change for $>4000 \mathrm{~s}$. The $I_{\mathrm{ON}} / I_{\mathrm{OFF}}(\mathrm{ON}$ and OFF current) ratio $\left(\sim 10^{6}\right)$ was high; this result confirms that the memory window was wide (Figure $6 \mathrm{c}$ ).

These results prove that this memory device fulfills the requirements of nonvolatile memory.

\section{Conclusions}

We studied the design and simulation of a simple MPA with perfect and broadband visible absorbance that depends on the phase of $\mathrm{Ge}_{2} \mathrm{Sb}_{2} \mathrm{Te}_{5}$ (GST). The total electrical and magnetic intensities as well as the related field distribution confirmed the physical origin of the absorption phenomenon. We also fabricated a nanoscale ReRAM device using GST and $\mathrm{Al}_{2} \mathrm{O}_{3}$ as insulator layers, and characterized the electrical properties. The memory device that uses GST and alumina as the bilayer solid electrolyte demonstrated reproducible bipolar switching properties. Furthermore, the fabricated ReRAM had a reliable performance with a high ON/OFF ratio $\left(\sim 10^{6}\right)$ and an acceptable data retention time $\left(>3 \times 10^{3}\right.$ $\mathrm{s})$. Based on the electrical measurements, the suggested conduction mechanism is Ohmic conduction and the switching mechanism is postulated to the formation and rupture of CFs.

Our proposed device is a potential candidate for optical storage systems, photonic synaptic devices, and energy-harvesting nanodevices.

Supplementary Materials: The following supporting information including a detailed description of experimental procedures, additional tables and supporting figures is available online at http://www.mdpi.com/2076-3417/9/ 3/564/s1.

Author Contributions: J.R. and N.R.-H. conceived the idea and initiated the project. N.R.-H. designed all the experiment, analyzed the data, fabricated samples, and wrote the manuscript. J.R. and N.R.-H. participated in the discussion, confirmed the achieved data, and approved the final manuscript. J.R. supervised the entire project.

Funding: This research was supported by the National Research Foundation of Korea (NRF) grants (NRF-2018M3D1A1058998, NRF-2015R1A5A1037668, CAMM-2019M3A6B3030637).

Conflicts of Interest: The authors declare no conflicts of interest.

\section{References}

1. Zhang, H.; Zhou, L.; Rahman, B.M.; Wu, X.; Lu, L.; Xu, Y.; Xu, J.; Song, J.; Hu, Z.; Xu, L.; et al. Ultracompact Si-GST hybrid waveguides for nonvolatile light wave manipulation. IEEE Photonics J. 2018, 10, 2200110. [CrossRef]

2. Boschker, J.E.; Lü, X.; Bragaglia, V.; Wang, R.; Grahn, H.T.; Calarco, R. Electrical and optical properties of epitaxial binary and ternary GeTe-Sb 2 Te 3 alloys. Sci. Rep. 2018, 8, 5889. [CrossRef]

3. Guo, Z.; Yang, X.; Shen, F.; Zhou, Q.; Gao, J.; Guo, K. Active-tuning and polarization-independent absorber and sensor in the infrared region based on the phase change material of Ge2Sb2Te5 (GST). Sci. Rep. 2018, 8, 12433. [CrossRef] [PubMed]

4. Chakraborty, I.; Saha, G.; Sengupta, A.; Roy, K. Toward fast neural computing using all-photonic phase change spiking neurons. Sci. Rep. 2018, 8, 12980. [CrossRef] [PubMed]

5. Wu, C.; Yu, H.; Li, H.; Zhang, X.; Takeuchi, I.; Li, M. Low-loss integrated photonic switch using sub-wavelength patterned phase change material. ACS Photonics 2019, 6, 87-92. [CrossRef] 
6. Raeis-Hosseini, N.; Lim, S.; Hwang, H.; Rho, J. Reliable Ge2Sb2Te5-Integrated high-density nanoscale conductive bridge random access memory using facile nitrogen-doping strategy. Adv. Electron. Mater. 2018, 4, 1800360. [CrossRef]

7. Li, X.; Youngblood, N.; Ríos, C.; Cheng, Z.; Wright, C.D.; Pernice, W.H.; Bhaskaran, H. Fast and reliable storage using a 5 bit, nonvolatile photonic memory cell. Optica 2019, 6, 1-6. [CrossRef]

8. Khan, M.N.I.; Jones, A.; Jha, R.; Ghosh, S. Sensing of phase-change memory. In Sensing of Non-Volatile Mmemory Demystified; Springer: Cham, Switzerland, 2019; pp. 81-102.

9. Babashah, H.; Kavehvash, Z.; Khavasi, A.; Koohi, S. Temporal analog optical computing using an on-chip fully reconfigurable photonic signal processor. Opt. Laser Technol. 2019, 111, 66-74. [CrossRef]

10. Rodriguez-Hernandez, G.; Hosseini, P.; Ríos, C.; Wright, C.D.; Bhaskaran, H. Mixed-mode electro-optical operation of Ge2Sb2Te5 nanoscale crossbar devices. Adv. Electron. Mater. 2017, 3, 1700079. [CrossRef]

11. Gu, M.; Li, X.; Cao, Y. Optical storage arrays: A perspective for future big data storage. Light-Sci. Appl. 2014, 3, e177. [CrossRef]

12. Rios, C.; Hosseini, P.; Taylor, R.A.; Bhaskaran, H. Color depth modulation and resolution in phase-change material nanodisplay. Adv. Funct. Mater. 2016, 28, 4720-4726. [CrossRef] [PubMed]

13. Midolo, L.; Schliesser, A.; Fiore, A. Nano-opto-electro-mechanical systems. Nat. Nanotechnol. 2018, $13,11$. [CrossRef] [PubMed]

14. Wuttig, M.; Yamada, N. Phase-change materials for rewriteable data storage. Nat. Mater. 2007, 6, 824-832. [CrossRef] [PubMed]

15. Wuttig, M. Phase-change materials: Towards a universal memory? Nat. Mater. 2005, 4, 265-266. [CrossRef] [PubMed]

16. Raeis-Hosseini, N.; Rho, J. Metasurfaces based on phase-change material as a reconfigurable platform for multifunctional devices. Materials 2017, 10, 1046. [CrossRef] [PubMed]

17. Au, Y.Y.; Bhaskaran, H.; Wright, C.D. Phase-change devices for simultaneous optical-electrical applications. Sci. Rep. 2017, 7, 9688. [CrossRef] [PubMed]

18. Feldmann, J.; Stegmaier, M.; Gruhler, N.; Ríos, C.; Bhaskaran, H.; Wright, C.D.; Pernice, W.H.P. Calculating with light using a chip-scale all-optical abacus. Nat. Commun. 2017, 8, 1256. [CrossRef] [PubMed]

19. Wright, C.D.; Hosseini, P.; Diosdado, J.A.V. Beyond von-Neumann computing with nanoscale phase-change memory devices. Adv. Funct. Mater. 2013, 23, 2248-2254. [CrossRef]

20. Hill, M.T.; Dorren, H.J.; De Vries, T.; Leijtens, X.J.; Den Besten, J.H.; Smalbrugge, B.; Oei, Y.S.; Binsma, H.; Khoe, G.D.; Smit, M.K. A fast low-power optical memory based on coupled micro-ring lasers. Nature 2004, 432, 206-209. [CrossRef]

21. Kim, R.H.; Kim, D.H.; Xiao, J.; Kim, B.H.; Park, S.I.; Panilaitis, B.; Ghaffari, R.; Yao, J.; Li, M.; Liu, Z.; et al. Waterproof AlInGaP optoelectronics on stretchable substrates with applications in biomedicine and robotics. Nat. Mater. 2017, 9, 929-937. [CrossRef]

22. Ríos, C.; Stegmaier, M.; Hosseini, P.; Wang, D.; Scherer, T.; Wright, C.D.; Bhaskaran, B.; Pernice, W.H.P. Integrated all-photonic non-volatile multi-level memory. Nat. Photonics 2015, 9, 725-732.

23. Loke, D.; Lee, T.H.; Wang, W.J.; Shi, L.P.; Zhao, R.; Yeo, Y.C.; Chong, T.C.; Elliott, S.R. Breaking the speed limits of phase-change memory. Science 2012, 336, 1566-1569. [CrossRef] [PubMed]

24. Ding, F.; Wang, Z.; He, S.; Shalaev, V.M.; Kildishev, A. Broadband high-efficiency half-wave plate: A supercell-based plasmonic metasurface approach. ACS Nano 2015, 9, 4111-4119. [CrossRef] [PubMed]

25. Yu, N.; Capasso, F. Flat optics with designer metasurfaces. Nat. Mater. 2014, 13, 139. [CrossRef] [PubMed]

26. Rios, C.; Hosseini, P.; Wright, C.D.; Bhaskaran, H.; Pernice, W.H. On-chip photonic memory elements employing phase-change materials. Adv. Mater. 2014, 26, 1372-1377. [CrossRef] [PubMed]

27. Hossain, M.J.; Faruque, M.R.I.; Islam, M.T.; Mat, K.B. A new compact octagonal shape perfect metamaterial absorber for microwave applications. Appl. Sci. 2017, 7, 1263. [CrossRef]

28. Luo, H.; Cheng, Y.Z. Ultra-thin dual-band polarization-insensitive and wide-angle perfect metamaterial absorber based on a single circular sector resonator structure. J. Electron. Mater. 2018, 47, 323-328. [CrossRef]

29. Krayer, L.J.; Kim, J.; Munday, J.N. Near-perfect absorption throughout the visible using ultra-thin metal films on index-near-zero substrates. Opt. Mater. Express 2019, 9, 330-338. [CrossRef]

30. Su, X.; Li, G.; Yang, H.; Zhao, Z.; Chen, X.; Lu, W. Dark mode driven extra-narrow and multiband absorber. Plasmonics 2018, 13, 729-735. [CrossRef] 
31. Sen, G.; Islam, S.N.; Banerjee, A.; Das, S. Broadband perfect metamaterial absorber on thin substrate for X-band and Ku-band applications. Prog. Electromagn. Res. 2017, 73, 9-16. [CrossRef]

32. Wu, C.; Shvets, G. Design of metamaterial surfaces with broadband absorbance. Opt. Lett. 2012, 37, 308-310. [CrossRef] [PubMed]

33. Huang, L.; Chowdhury, D.R.; Ramani, S.; Reiten, M.T.; Luo, S.N.; Taylor, A.J.; Chen, H.T. Experimental demonstration of terahertz metamaterial absorbers with a broad and flat high absorption band. Opt. Lett. 2012, 37, 154-156. [CrossRef] [PubMed]

34. Michel, A.K.U.; Wuttig, M.; Taubner, T. Design parameters for phase-change materials for nanostructure resonance tuning. Adv. Opt. Mater. 2017, 5, 1700261. [CrossRef]

35. Lee, D.; Jeong, H.; Lim, S. Electronically switchable broadband metamaterial absorber. Sci. Rep. 2017, 7, 4891. [CrossRef] [PubMed]

36. Li, W.; Valentine, J. Metamaterial perfect absorber based hot electron photodetection. Nano Lett. 2014, 14, 3510-3514. [CrossRef] [PubMed]

37. Cao, T.; Wei, C.-W.; Simpson, R.E.; Zhang, L.; Cryan, M.J. Broadband polarization-independent perfect absorber using a phase-change metamaterial at visible frequencies. Sci. Rep. 2014, 4, 3955. [CrossRef] [PubMed]

38. Driscoll, T.; Kim, H.T.; Chae, B.G.; Kim, B.J.; Lee, Y.W.; Jokerst, N.M.; Palit, S.; Smith, D.R.; Di Ventra, M.; Basov, D.N. Memory Metamaterials. Science 2009, 325, 1518-1521. [CrossRef] [PubMed]

39. Zheludev, N.I.; Kivshar, Y.S. From metamaterials to metadevices. Nat. Mater. 2012, 11, 917-924. [CrossRef]

40. Raeis Hosseini, N.; Lee, J.-S. Resistive switching memory based on bioinspired natural solid polymer electrolytes. ACS Nano 2014, 9, 419-426. [CrossRef]

41. Raeis-Hosseini, N.; Lee, J.-S. Resistive switching memory using biomaterials. J. Electroceram. 2017, 39, 223-238. [CrossRef]

42. Wuttig, M.; Bhaskaran, H.; Taubner, T. Phase-change materials for non-volatile photonic applications. Nat. Photonics 2017, 11, 465-476. [CrossRef]

43. Shportko, K.; Kremers, S.; Woda, M.; Lencer, D.; Robertson, J.; Wuttig, M. Resonant bonding in crystalline phase-change materials. Nat. Mater. 2008, 7, 653-658. [CrossRef] [PubMed]

44. Cao, T.; Zhang, L.; Simpson, R.E.; Cryan, M.J. Mid-infrared tunable polarization-independent perfect absorber using a phase-change metamaterial. J. Opt. Soc. Am. B 2017, 30, 1580-1585. [CrossRef]

45. Liu, W.; Kivshar, Y. Multipolar interference effects in nanophotonics. Philos. Trans. Royal Soc. A 2017, 375 , 20160317. [CrossRef] [PubMed]

46. Wei, L.; Miroshnichenko, A.E.; Kivshar, Y.S. Control of light scattering by nanoparticles with optically-induced magnetic responses. Chin. Phys. B 2014, 23, 047806.

47. Kerker, M.; Wang, D.-S.; Giles, C. Electromagnetic scattering by magnetic spheres. J. Opt. Soc. Am. 1983, 73, 765-767. [CrossRef]

48. Aydin, K.; Ferry, V.E.; Briggs, R.M.; Atwater, H.A. Broadband polarization-independent resonant light absorption using ultrathin plasmonic super absorbers. Nat. Commun. 2011, 2, 517. [CrossRef] [PubMed]

(c) 2019 by the authors. Licensee MDPI, Basel, Switzerland. This article is an open access article distributed under the terms and conditions of the Creative Commons Attribution (CC BY) license (http://creativecommons.org/licenses/by/4.0/). 